\title{
El final de la Avenida Valencia al Mar
}

\section{Ciro Vidal Climent ${ }^{1}$, Ivo Vidal Climent ${ }^{2}$, Vicente Vidal Vidal ${ }^{3}$}

Departamento de proyectos arquitectónicos, Universitat Politècnica de València.

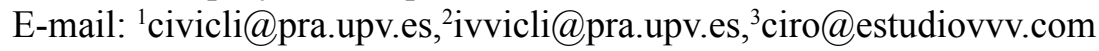

\begin{abstract}
Resumen. El paseo de Valencia al Mar afronta desde hace 20 años un debate sobre la pertinencia de su prolongación. Actualmente la avenida llega hasta la estación pasante del Cabañal, de manera que el conjunto urbano que contacta con el mar es el poblado marítimo. Pero el poder de la semántica es ajeno a la realidad física y la inevitabilidad asociada al dicho de que todo llega al mar empuja a una traducción literal de continuar el paseo desoyendo los precisos argumentos sobre la morfología urbana y la irracionalidad del destructivo corte de la trama lineal del Cabanyal. Para encauzar la explicación de una propuesta alternativa se hace una lectura histórica del paseo de Blasco Ibáñez desde que lo proyectara el arquitecto Casimiro Meseguer a finales del siglo XIX como una avenida ajardinada de $100 \mathrm{~m}$ de anchura delimitada por viviendas unifamiliares que partía del jardín de Viveros (antiguo palacio Real) hasta llegar al Cabañal. También se estudia el plan parcial no ejecutado que en 1960 hizo Fernando Moreno Barberá atravesando el poblado marítimo.

La propuesta que se presenta consiste en entender que la vinculación con el mar se puede producir a través de una promenade ajardinada situada en el eje central del paseo desde la que se puede divisar el mar debido a la escasa altura del caserio del poblado marítimo. Este final en ladera, con una suave pendiente del 3\%, nos conduce a una cota elevada con la que se puede cruzar el Bulevar Serrería, acceder a un intercambiador modal situado sobre la nueva cubierta de la estación y llegar al lado sureste del bulevar, configurando alli un centro direccional formado por una plataforma paralela al bulevar que sustenta cuatro torres de viviendas y adquiere una mixtura de usos que activará la zona.
\end{abstract}

Palabras clave: Promenade; intercambiador modal; plataforma; estación pasante; poblado marítimo

\section{Introducción histórica}

El trazado del Paseo Valencia al Mar, denominado Avenida de Blasco Ibáñez, se acota entre los jardines del palacio Real al norte y el Poble Nou del Mar al sur conformado por el Cabañal, el Cañamelar y el Cap de França. El proyecto de 1888 redactado por Casimiro Meseguer tomó el nombre de Paseo de Valencia al Mar que era como decir hasta el Poblado Nuevo del Mar, cuyo municipio era independiente y sobre el que Valencia no tenía jurisdicción. En el plano del año 1899 (figura 1)que cerraba el siglo XIX Meseguer rebautizó su trazado con el nombre de Paseo de Valencia al Cabañal, barrio que ya formaba parte de la ciudad de Valencia, pues en el año 1897 la jurisdicción de los poblados marítimos había sido transferida a la ciudad de Valencia.

Algunas precisiones sobre los modos y las formas.

La traza de Meseguer incluía tres bandas de 100 metros cada una, de las cuales las dos laterales se dedicaban a edificación y la central abarcaba las vías rodadas y aceras generosas jalonadas con plátanos, además del paseo ajardinado con un centro levemente deprimido donde crecía un arbolado interior de cuatro en fondo. Las vías rodadas con sus aceras laterales servían al desarrollo de la Ciudad jardín como 


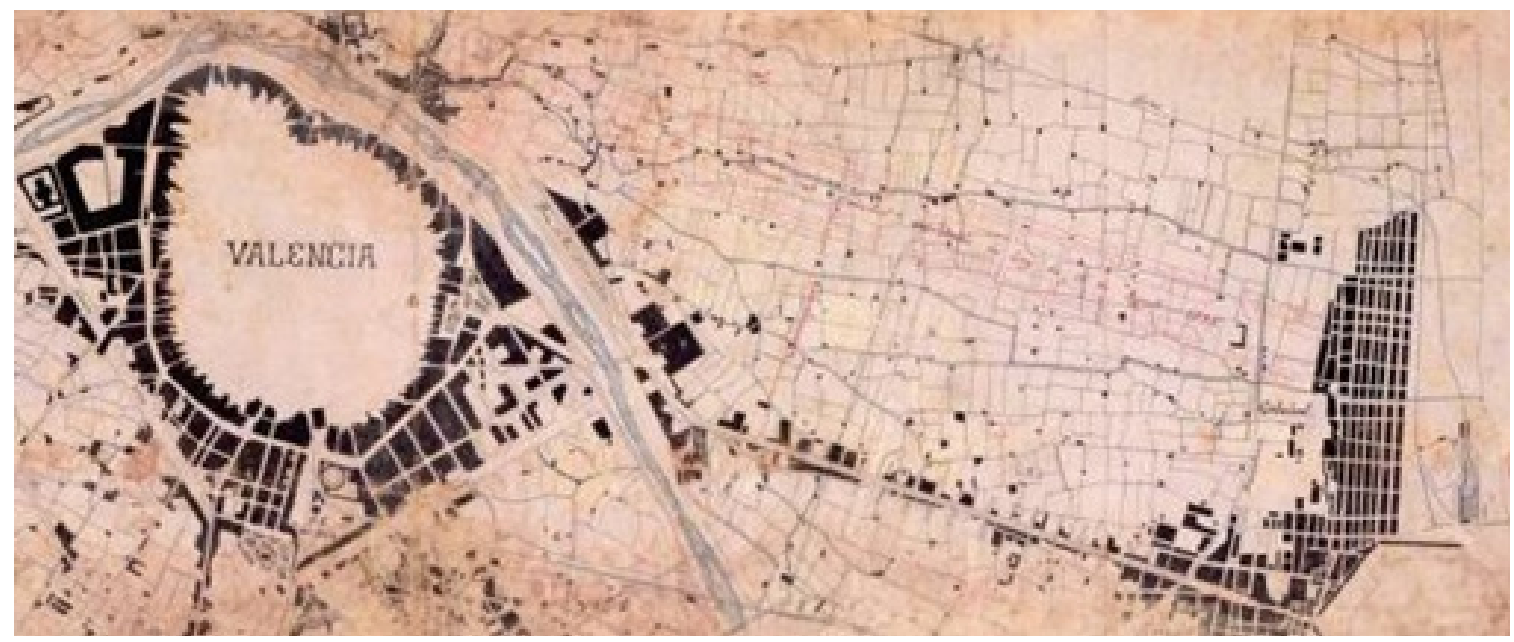

Fig. 1. Casimiro Meseguer. Plano del Paseo Valencia al Cabañal, 1899.

un ideal de inspiración burguesa cuyo recorrido llegaba justamente hasta el Cabañal. Con esta sección transversal solo se construyeron al inicio, ocupando la zona del noreste, veinte chalets que Enrique Viedma proyectó para la asociación de la prensa valenciana en 1931. $\mathrm{Su}$ persistencia en la actualidad, junto con la urbanización del jardín central, nos informa fielmente de la gran calidad de ese trazado primigenio.

En las dos bandas laterales de este primer sector del norte, después de la parcelación de los periodistas, fueron ubicándose los edificios de la Universidad de Valencia como el observatorio astronómico junto a la facultad de medicina. El trazado de estos proyectos corresponde al año 1908, siendo desarrollada la facultad de medicina entre los años 1920 y 1949 según el proyecto de Alfonso Fugairiño y Luis Albert, a la vez que el observatorio de Mariano Peset Aleixandre construido entre 1935 y 1944. Estos proyectos, junto con el colegio mayor Luís Vives, fueron el embrión de la ciudad universitaria que el rector Juan Peset Aleixandre impulsó desde los años 30 como una decidida apuesta por la modernidad para desarrollar un programa complejo que implicó la transformación de la ciudad jardín propuesta por Meseguer en un área universitaria afín a la expansión de la ciudad de Valencia.

Los nuevos edificios de Fernando Moreno Barberá, como las facultades de Derecho, de Historia, de Filosofía y la Escuela de Ingenieros Agrónomos, formaron el campus del Paseo al Mar como ámbito de expansión de la Universidad de Valencia. Además, los edificios públicos para sedes administrativas, cuyo concurso ganó Miguel Colomina Barberá, acabaron por consolidar el carácter institucional del Paseo entre el jardín de los Viveros y la Avenida de Aragón, que fue el resultado de la eliminación del trazado de ferrocarril de Valencia a Zaragoza.

A finales de los años 50 el Ayuntamiento de Valencia convocó un concurso para la contratación de arquitectos con el objetivo de desarrollar el planeamiento parcial de varias zonas del Plan General de Valencia y su cintura, redactado en 1946. En el mes de septiembre de 1959 se le adjudicó a Moreno Barberá el Plan Parcial $n^{\circ} 13$ (figura 2) que consistía en la prolongación de la Avenida de Blasco Ibáñez a través del Cabañal hasta llegar a la playa, con dos espacios subsidiarios formados por dos bandas laterales a lo largo de su eje formados por ordenaciones volumétricas de bloques lineales que reflejaban la admiración por las modernas ordenaciones urbanísticas de preguerra del norte de Europa, o los edificios Hötorget que se estaban construyendo en Estocolmo en ese momento, pues el tramo central muestra en cada banda cinco edificios de 16 plantas situados longitudinalmente a la avenida como la manera de delimitar volumétricamente la trama del poblado marítimo. Este Plan Parcial que desarrollaba la prolongación hasta el mar no llegó a construirse, sin embargo la expansión noreste de la ciudad tomó forma gracias a la formación de un verdadero distrito universitario a ambos 


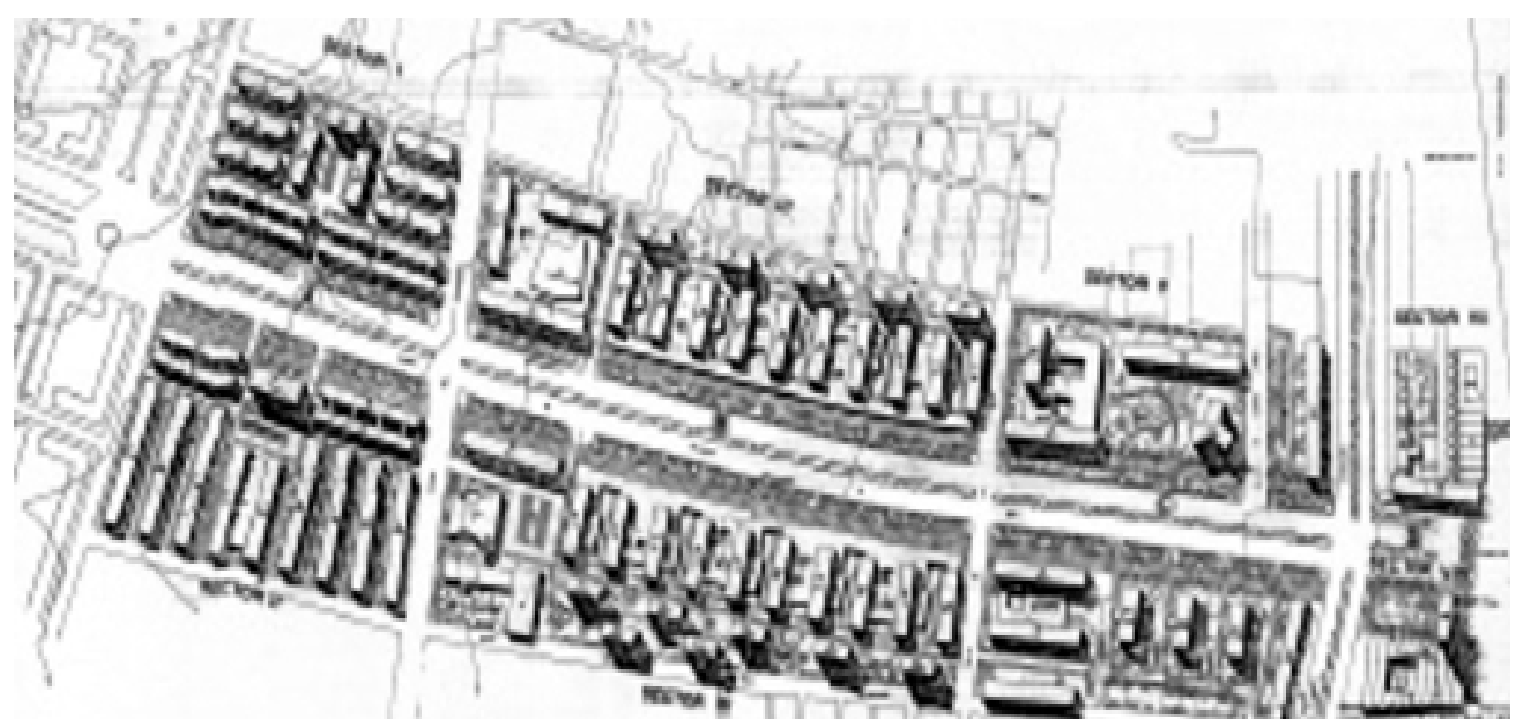

Fig. 2. Fernando Moreno Barberá. Plan Parcial no 13.

lados de la Avenida de los Naranjos, con la Universitat Politècnica de nueva creación, y la expansión de la Universidad de Valencia.

\section{Actualidad}

En 1998 el consistorio de la alcaldesa Rita Barberá presentó un plan urbanístico para la prolongación en línea recta de la avenida Valencia al Mar que implicaba la demolición de más de 400 inmuebles del barrio marinero, una parte de los cuales ocupaban una zona que en 1993 había sido declarada bien de interés cultural (BIC). En 2010 el Ministerio de Cultura emitió una orden que protegía el barrio del Cabañal de las demoliciones ordenadas de modo que se frenó la destrucción del barrio. En 2014 se llegó a un preacuerdo entre el Ayuntamiento y el Ministerio para desbloquear el Plan del Cabañal con un rediseño de la prolongación que disminuía en una tercera parte el número de inmuebles afectados al adaptarse a la angulación propia de la retícula histórica del poblado y al reducirse a la mitad el ancho del paseo.

Actualmente la solución final de la Avenida Valencia al Mar está todavía pendiente de conclusión. Los límites del histórico barrio del Cabañal se interponen en la obsesiva marcha de la avenida hacia el Mediterráneo, por lo que el debate sobre su prolongación definitiva continúa estando presente como una pugna política en la que se ha mantenido constante la firme oposición de los vecinos. Estos se han organizado a través de diferentes iniciativas ciudadanas, apoyados por un colectivo profesional y docente con cierto rigor teórico cuyas propuestas programáticas han permitido elevar el problema a instancias judiciales, pero el debate continúa sin cerrarse. La propuesta que se presenta en este escrito se realiza para ampliar las opciones propositivas que puedan ayudar a vislumbrar otra posible solución al conflicto.

\section{Hötorget, un caso de estudio}

Los cinco edificios Hötorget de Estocolmo (Suecia) están situados entre las plazas Hötorget y Sergels Torg. Fueron una apuesta singular por la arquitectura moderna en un centro urbano consolidado y no destruido por la Segunda Guerra Mundial. La propuesta inicial de 1946 es del arquitecto David Helldén. Posteriormente, en 1951, junto a Sven Markelius, empezaron a desarrollar el proyecto urbanístico de los edificios y alrededores en el que se añadieron los puentes peatonales y se aumentó la altura de los edificios a 19 plantas mientras que se redujo la de los alrededores a dos plantas, configurando una ordenación volumétrica muy clara (figura 3). Esta disposición se acercó aún más a la voluntad original de renovar el centro de la ciudad mediante los principios de una arquitectura volumétricamente limpia, exenta y de aspecto cristalino gracias a los 


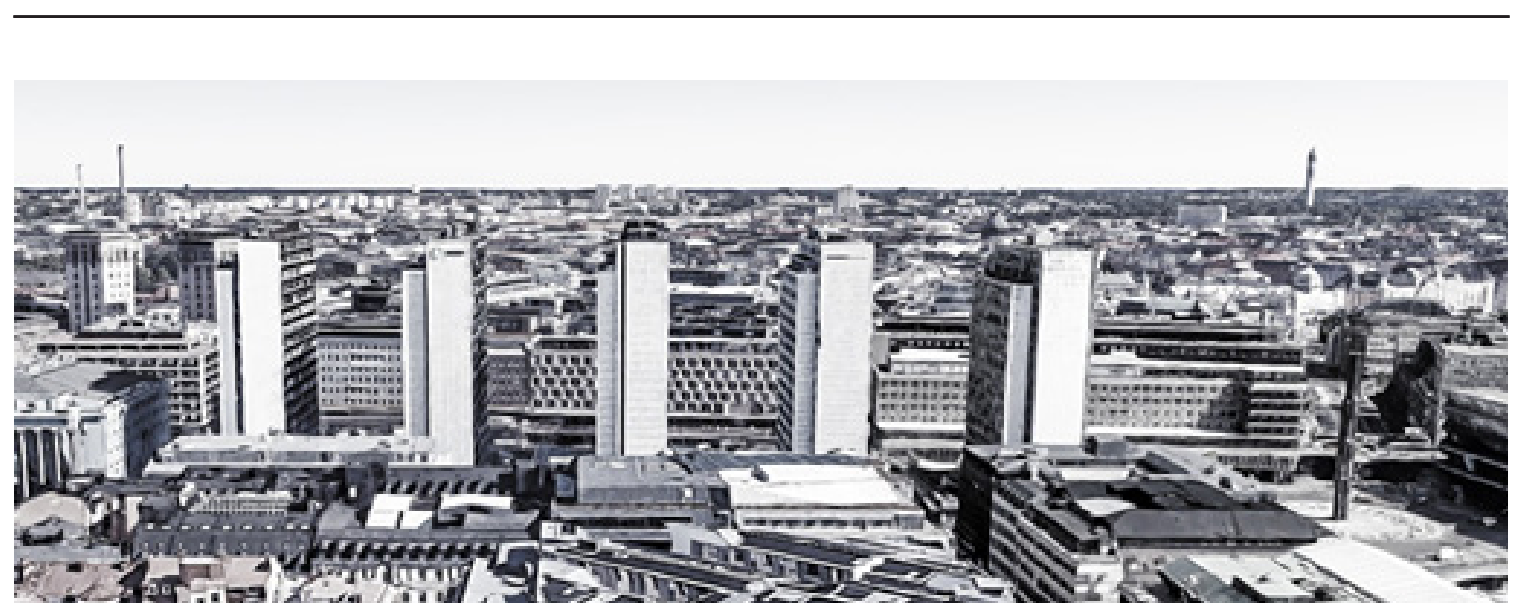

Fig. 3. Conjunto de edificios Hötorget, Estocolmo (Suecia).

muros cortina, una solución poco empleada en Suecia pero que tenía el cercano referente de la Lever House de Skidmore, Owings y Merrill, concluida en 1952.

Los edificios Hötorget fueron construidos entre 1952 y 1966 por distintos arquitectos y fueron un ejemplo muy real de la capacidad regenerativa de la arquitectura moderna dentro de tejidos históricamente consolidados. Demostraba que la miscibilidad de estructuras urbanas racionales era posible y aconsejable pues, a la vez que dotaba de identidad a una zona de la ciudad, respondía a las legítimas aspiraciones higienistas de iluminación y ventilación, al rigor del orden geométrico, a la necesidad de espacios verdes y abiertos, y a la voluntad de una presencia volumétrica que no solo es foco de las vistas, sino que permite nuevas visiones sobre la ciudad y el entorno. En definitiva, una muestra de proyecto urbano de arquitectura contemporánea implementado con elegancia y cierto anonimato en una ciudad de amplio relieve histórico.

\section{Propuesta urbana}

De los cruces de calles vinculados a los distintos ambientes que las calles transversales provocaban se ha mantenido a través del tiempo el Bulevar Serrería, el último que atraviesa la avenida de Blasco Ibáñez y que marca un límite claro al sur, puesto que es el resultado de eliminar el trazado en superficie de la antigua línea de ferrocarril Valencia a Tarragona. Este Bulevar mantiene en su trazado el ferrocarril subterráneo que sustituye al anterior en superficie y en su proyección de futuro debe adquirir relevancia como el lugar para afianzar una estación pasante del eje mediterráneo. Esta infraestructura ferroviaria es necesaria para el desarrollo de la ciudad capitalina en el conjunto del arco mediterráneo, pero debe asumir la compleja delimitación del poblado marítimo del Cabañal dentro de la estructura de la ciudad, revalorizando un área urbana de gran atractivo morfológico y posición frente al litoral.

La estación pasante y los servicios que trae consigo pertenecen al material urbano capaz de dotar al conjunto de la ciudad de la suficiente."sinoikismos" aristotélica, cuya voz moderna "sinergia", ayuda a entender que, como las ecuaciones de Strogartz, la ciudad es un todo mayor que la suma de sus partes. La solución por partes es un recurso necesario para resolver las incógnitas de la ciudad a la manera de los complejos sistemas de ecuaciones lineales, por tanto tenemos que dividir la ciudad por piezas y resolverlas por separado recombinando finalmente las respuestas aisladas para mantener la unidad morfológica, cuya precisa expresión es la razón de ser de un urbanismo culto.

La ciudadestá continuamentereformándosey para favorecer la operatividad de sus problemas exige estudiar sus piezas integrándolas cada vez con su entorno, piezas como las que componen el nudo urbano que se forma en la intersección del Bulevar Serrería y la avenida de Blasco Ibáñez (figura 4). Una propuesta de futuro para un área metropolitana de envergadura debe ir más allá de las apresuradas soluciones 


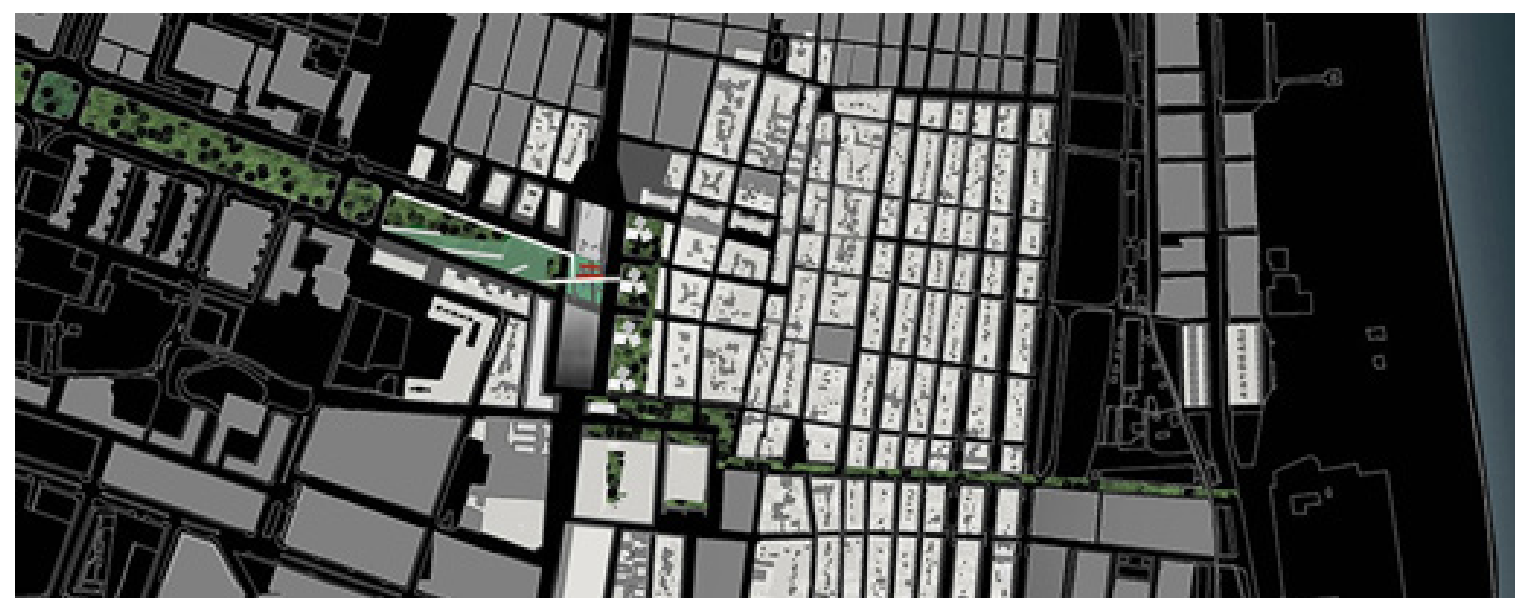

Fig. 4. Planta general de la propuesta del nudo final de Blasco Ibáñez.

de compromiso que, intentando contentar a todos los intereses particulares, obvia el interés general de la ciudad.

El continuo ajardinado central del Paseo Valencia al Mar se modifica con un último tramo configurado por una ladera del $3 \%$ de pendiente cuya tensión produce la elevación de una plataforma ajardinada que pasa por encima del Bulevar Serrería formando un suave prado en una cota donde las vistas van ganando en profundidad hasta llegar a un nivel donde se divisa el mar (figura5).

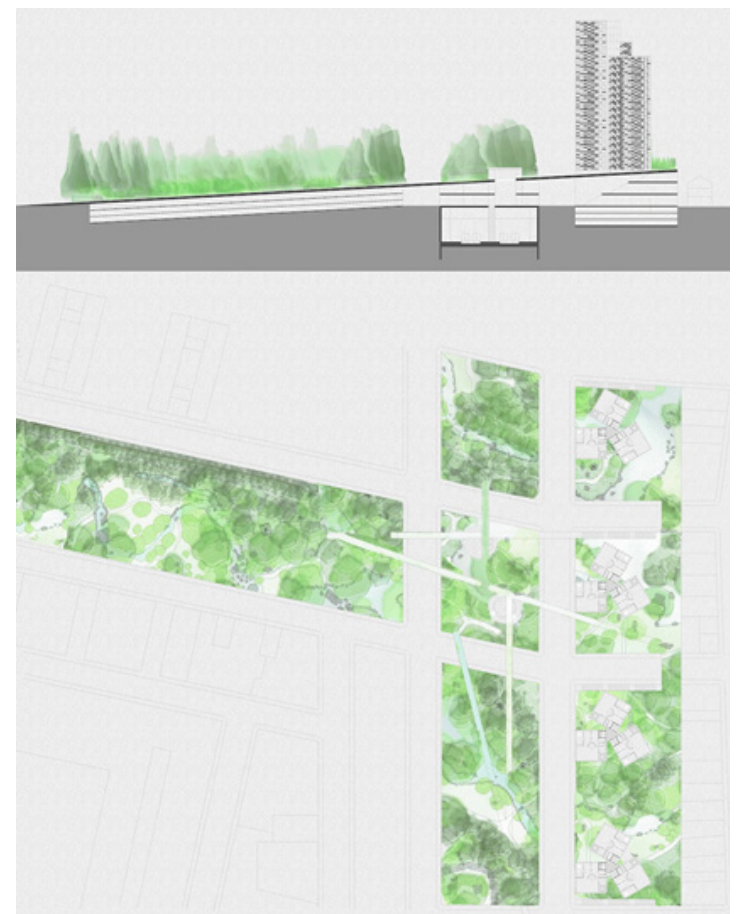

Fig. 5. Planta y sección de la promenade final de Blasco Ibáñez.
Bajo el plano inclinado que conduce a la plataforma elevada se sitúa un aparcamiento de vehículos con un $1 \%$ de pendiente capaz de albergar un millar de automóviles, lo cual permite un uso más versátil del intercambiador modal de la estación y libera el litoral de una gran carga de presión rodada.

La estación pasante en el Bulevar Serrería resuelve un nudo crucial en la ciudad moderna a la vez que el flujo de visitantes ofrece la ventaja de incorporar nuevas actividades como un terciario potente y el atractivo de nuevas viviendas con vistas al mar y cercanas al conjunto universitario como áreas de gran demanda y futuro. La solución arquitectónica propuesta persigue la integración de un vecindario cercano perteneciente a los poblados marítimos, con el resto del área metropolitana en la que prevalece la actividad de la Universitat Politècnica de València con el gran dinamismo de la docencia y los laboratorios de investigación.

La estación pasante propiamente dicha tiene un desarrollo lineal de cuatro vías y tres andenes con una longitud de medio kilómetro $\mathrm{y}$ ofrece una gran permeabilidad transversal, así como accesibilidad a los distintos tipos de transporte y movilidad urbana mediante un intercambiador modal pensado para abastecer tanto el área interurbana como la de cercanías vinculada a la enseñanza y al trabajo zonal en los Institutos de Investigación y una potente dotación de terciario. 


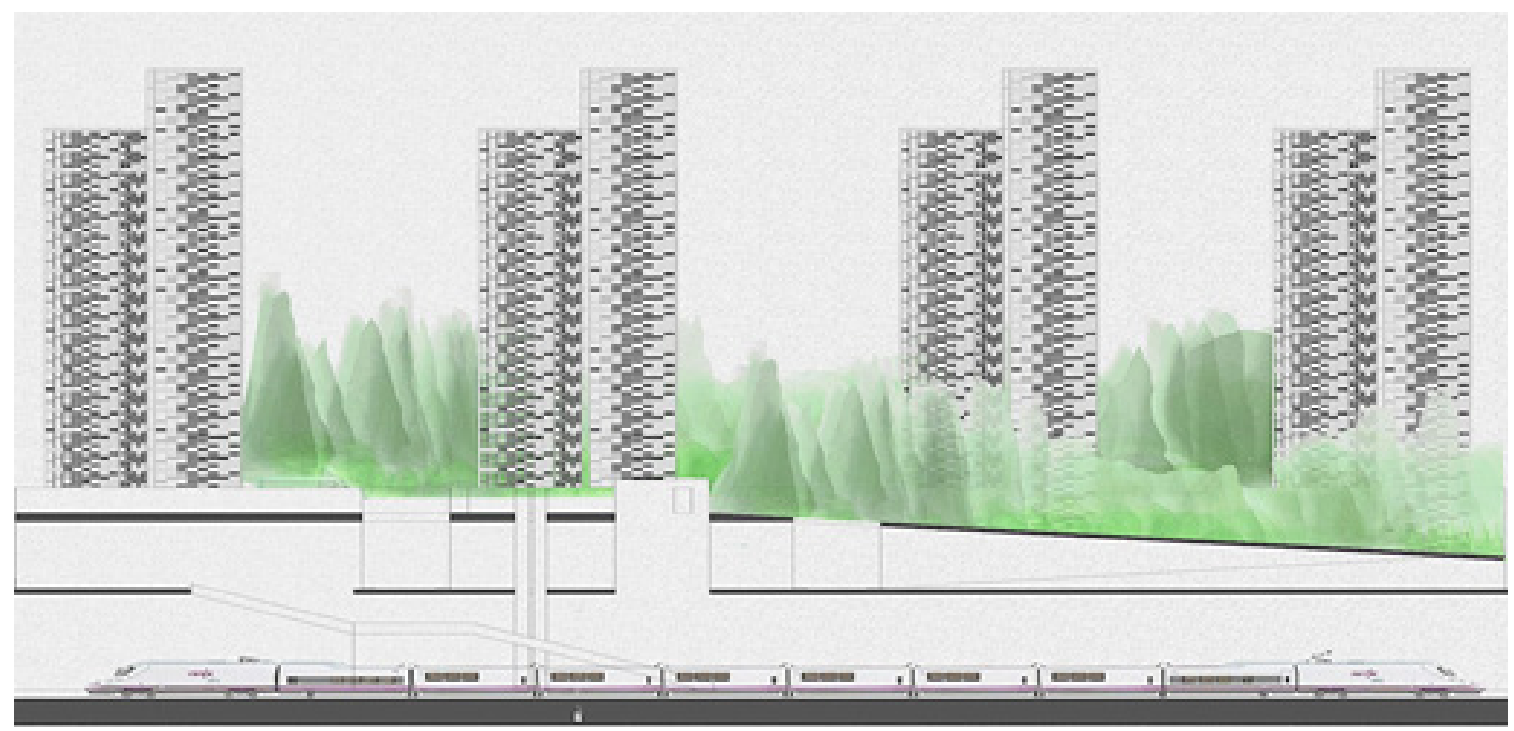

Fig. 6. Sección por estación y alzado de la plataforma final de Blasco Ibáñez.

Una vez atravesado el Bulevar Serrería queda estabilizado un plano horizontal elevado y ajardinado sobre el que se proponen cuatro torres de viviendas y bajo el que se desarrolla todo el programa terciario. Esta plataforma (figura 6), que acompaña en su trayecto al Bulevar Serrería, proporciona vistas por encima del caserío del Cabañal y acepta profundidades visuales del litoral marítimo. Sobre este espacio neutro y continuo se asienta el conjunto de cuatro torres, cada una de ellas formada por tres prismas de base $12 \times 12 \mathrm{~m}$ desplegados alrededor de un núcleo triangular y equilátero de $5 \mathrm{~m}$ de lado cuya transparencia fenomenológica proporciona visualmente el final de la avenida de Valencia al Mar, pues desarrolla sin opacidad un conjunto de escala y medida afín a la ciudad en su componente litoral. El acceso a la plataforma que está a la altura de tres plantas comerciales, se realiza a través de un nuevo paseo urbano utilizable tanto por los vecinos que salen a dar una vuelta como a los turistas que sienten la curiosidad que suscita la singularidad de esta ordenación (figura 7). Las vistas de largo alcance sobre el litoral y el recorrido a través de pasos elevados separados del tráfico urbano también nos remiten a los puentes de Vaux en los jardines de Olmsted del parque central neoyorquino.

Los núcleos de comunicación que soportan las cuatro torres emergen de una plataforma que mantiene el acceso público en su superficie ajardinada al mismo nivel donde se ubicarán los comercios de los servicios distribuidos. El desarrollo de los prismas de viviendas de 12 $\times 12 \mathrm{~m}$ alrededor de su núcleo equilátero de 6 $\mathrm{m}$ de lado, muestra la solución constructiva de un tipo de fachadas exentas y de planta libre, cuyas vistas al litoral las hace especialmente atractivas para una población cuyas

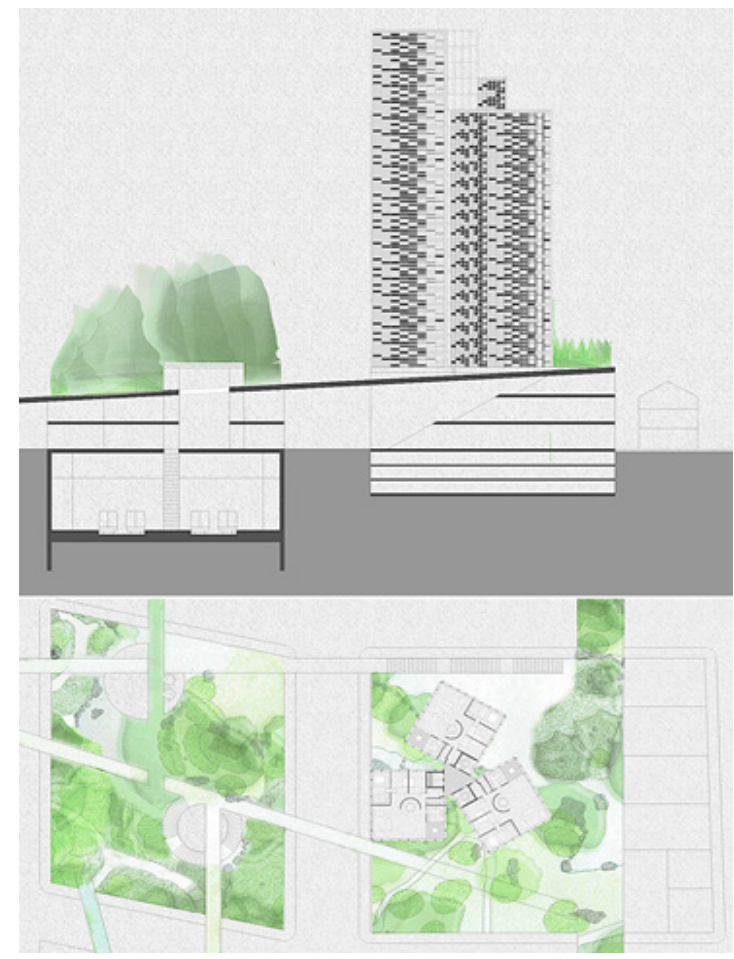

Fig. 7. Planta y sección de la estación y plataforma final de Blasco Ibáñez. 
aspiraciones familiares son homologables con las viviendas del Cabañal pero cuyos usuarios prefieren un orden vertical, como elección que valora las vistas por encima de la bondad de la calle.

Las plantas que soportan la plataforma a 15 $m$ de altura y en tantas otras bajo el nivel de la calle se reúnen las plantas destinadas a los servicios distribuidos que en forma de galería o bien centro de comercio dotan a la zona de una gran miscibilidad, pues su conexión con la estación pasante favorece la heterogeneidad que sociológicamente resulta pertinente para el lugar, la inversión y el respeto por la atmósfera de los poblados marítimos que quedan salvaguardados por el carácter de final singular que aporta a la Avenida de Blasco Ibáñez. Esta solución urbanística y arquitectónica acepta la sutura que se produce en la discontinuidad de los tramos como una propuesta de continuidad urbana en vez de la ruptura temida por la aplicación de medidas cuya arquitectura inmediata en tiempos de bonanza no hubiera soportado el paso del tiempo histórico. Esta intervención despejaría de los continuos avatares una zona tan peculiar y delicada como el Cabañal, que podría, por fin, consolidar la gran calidad morfológica que la continuidad de sus calles ofrecen.

\section{Conclusiones}

Esta propuesta trata de hacer verosímil la capacidad de la arquitectura para conciliar intereses aparentemente contrapuestos. Por un lado, pone de manifiesto la fortaleza geométrica de la trama del Cabañal, es decir, cuenta con ella como aliada para asumir que la forma de contacto entre la ciudad y la costa ya está adecuadamente resuelta. Por otro, evidencia el inapropiado final de la avenida de Blasco Ibáñez, lo cual es el aspecto menos debatido, pero no menos espinoso, pues la prolongación hasta el mar no le proporciona a la avenida un final digno, sino que deja irresuelta su agónica necesidad de completitud. La propuesta además incide en otros aspectos que se ha dejado de lado en el debate y que son de primera magnitud, como la importancia de la estación del Cabañal como un nudo vertebrador del eje de Alta Velocidad del arco mediterráneo, con una capacidad de tener mayor relevancia geopolítica, acorde con las últimas propuestas que se están estudiando en Madrid como el proyecto de convertir Atocha en estación pasante de Alta Velocidad.

La ordenación volumétrica de las cuatro torres produce un final diafragmático de la Avenida de Blasco Ibáñez que lo acota con precisión entre el Jardín de Viveros y la estación del Cabañal. Del mismo modo, desde

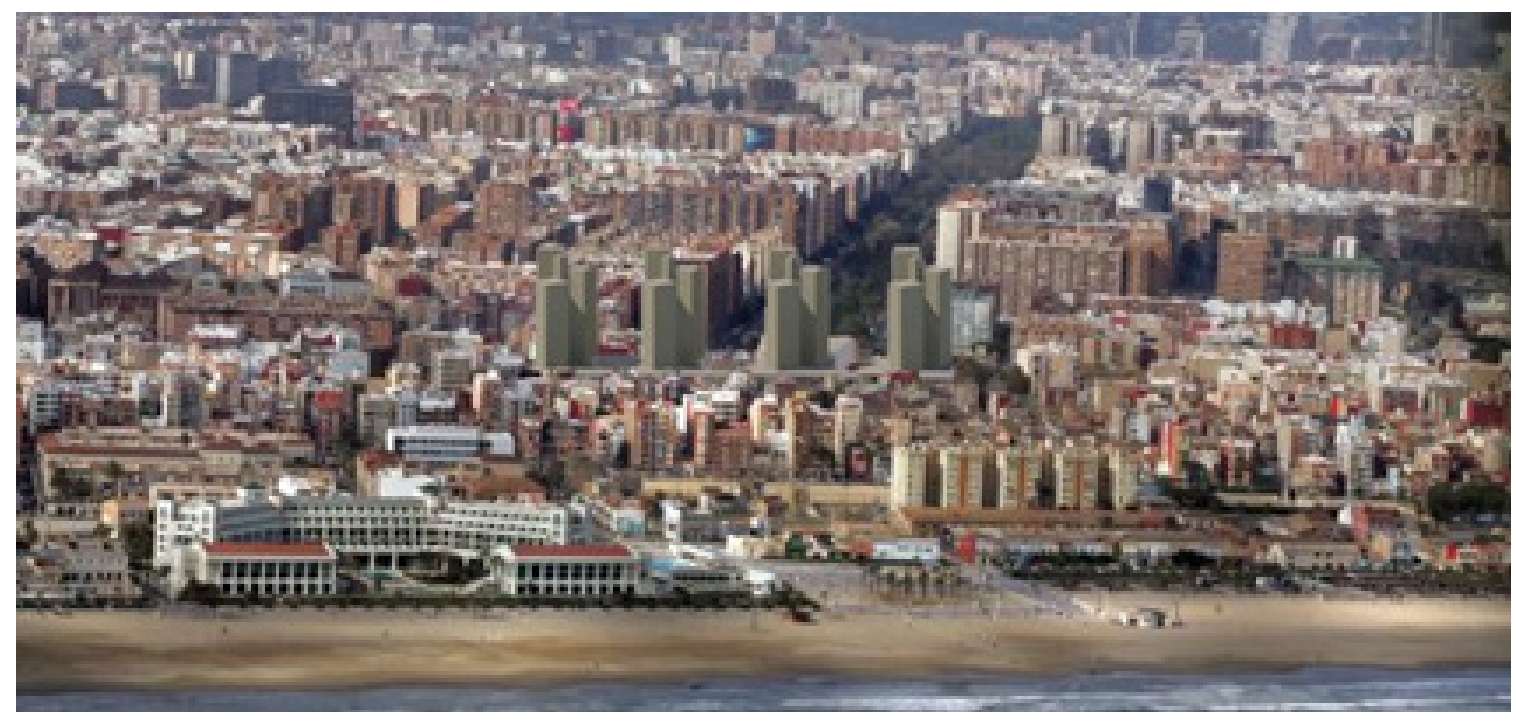

Fig. 8. Vista aérea desde el mar de la volumetría propuesta. 
el mar, la regular disposición de vacíos entre la emergencia de las torres (figura 8) posiciona ese final de la avenida y ese límite del poblado marítimo, actualizando el perfil noreste de la ciudad y otorgándole una reconocible y sugerente identidad acorde con este nuevo siglo.

\section{Referencias}

Bravo, Juan. 2016. "Fernando Moreno Barberá y la configuración de la Avenida de Blasco Ibáñez". En Valencia 1808-2015 La historia continua Vol I: 115-51. Valencia: Balandra edicions. ISBN 978-84-943874-8-7

Piñón, Juan Luis. 1988. "Razones y sinrazones del Paseo Valencia al Mar de Casimiro Meseguer", pp1.3.1-1.3.14, en Vol II: Siglos XIX y XX, en I Congrès d'història de la Ciutat de València, $3 \mathrm{~V}$, Valencia. Ajuntament de València 1988. 